\title{
Evaluación de la Pérdida de Calidad Mecánica de Tres Fibras Naturales como Alternativa de Tutorado en el Cultivo de Arveja (Pisum sativum L.)
}

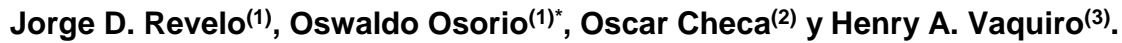 \\ (1) Facultad de Ingeniería Agroindustrial, Universidad de Nariño, Grupo de apoyo a la Investigación y Desarrollo \\ Agroalimentario GAIDA, Colombia (e-mail: jormilros@hotmail.com; osorio_oswaldo@udenar.edu.co) \\ (2) Facultad de Ingeniería Agronómica, Universidad de Nariño, Grupo de Investigación en Cultivos Andinos, GRICAND, \\ Colombia (e-mail: gricandudenar@gmail.com) \\ (3) Facultad Ingeniería Agronómica, Universidad del Tolima, Grupo de Investigación Centro de Desarrollo Agroindustrial \\ del Tolima Cedagritol, Colombia (e-mail: havaquiro@ut.edu.co)
}

Recibido Oct. 30, 2018; Aceptado Ene. 4, 2019; Versión final Abr. 6, 2019, Publicado Oct. 2019

\begin{abstract}
Resumen
El objetivo de este estudio fue determinar la velocidad de la pérdida de calidad mecánica de las propiedades de carga máxima, módulo de Young y elongación de fibras naturales de fique, algodón y plátano, al ser usadas como soporte vertical en el cultivo de arveja. La pérdida de calidad de las propiedades de las fibras naturales fue medida cada 15 días durante un periodo de tres meses en condiciones de cultivo. Los resultados fueron comparados con la fibra de polipropileno usada actualmente en estos sistemas de producción agrícola. La cinética de degradación de cada propiedad mecánica fue modelada matemáticamente y se estimaron parámetros cinéticos para cada fibra evaluada. La fibra de plátano presentó mayores velocidades de pérdida de calidad mecánica en la mayoría de las propiedades evaluadas y podría descartarse para su uso en el tutorado de arveja. Sin embargo, las fibras de fique y algodón presentaron menor velocidad con pérdida de calidad similar, y podrían utilizarse para sustituir a la fibra de polipropileno en los sistemas de producción de este tipo de cultivos agrícolas.
\end{abstract}

Palabras clave: calidad mecánica; fibras naturales; cultivos agrícolas; arveja; Pisum sativum L.).

\section{Evaluation of the Loss of Mechanical Quality of Three Natural Fibers as Alternative Supports in the Agricultural Production of Pea (Pisum sativum L.)}

\begin{abstract}
The objective of this study was to determine the quality loss rate of the mechanical properties maximum load, Young's modulus, and elongation at break of natural fibers of fique, cotton, and banana stalk, which were used as vertical supports in pea production. The quality loss of natural fiber properties was determined every 15 days for three months in crop conditions. The results were compared with those for polypropylene fiber, currently used in these agricultural production systems. The degradation kinetics for each mechanical property were mathematically modeled, and the kinetic parameters for each fiber were estimated. The banana-stalk fiber showed higher quality loss rates in most of the mechanical properties, and it could be discarded as vertical support in pea crop. However, the cotton and fique fibers presented lower and similar quality losses, and they could be used as an alternative to polypropylene in production systems of this type of crops.
\end{abstract}

Keywords: mechanical quality; natural fibers; agricultural production; pea; Pisum sativum L. 


\section{INTRODUCCIÓN}

La arveja (Pisum sativum L.) es una leguminosa de gran importancia en Colombia y en especial en el sur de la región de Nariño. Aunque su consumo se da principalmente en fresco y deshidratado, se han desarrollado proyectos de investigación que tienden a presentar alternativas para este producto como enlatados, snacks entre otras presentaciones que manifiestan su potencialidad en cuanto a industrialización se refiere (Pantoja et al., 2016), La siembra de arveja en el departamento de Nariño se realiza mediante un sistema denominado tutorado tradicional que es capaz de soportar los tallos trepadores de esta leguminosa permitiendo que produzcan una mayor cosecha (Forero y Ligarreto, 2009). El cultivo tiene un ciclo de vida a partir de la siembra, así: floración a los 75 días, cosecha de grano verde a los 120 días y cosecha grano seco a los 170 días (DANE, 2015). En este tutorado se emplean fibras sintéticas de polipropileno que están provocando problemas de contaminación para los agricultores, debido a que se requiere el uso de aproximadamente $225.000 \mathrm{~m}$ de fibra por cada hectárea de arveja tutorada verticalmente, los cuales ocasionan gastos de limpieza y reciclaje después de su uso, y en algunas ocasiones, problemas de aglomeración al caer al suelo, con las consecuentes dificultades durante la mecanización agrícola o alimentación de animales que pastan en los terrenos en época de descanso.

La alta resistencia a la corrosión, al agua y a la descomposición bacteriana convierte a las fibras sintéticas en residuos difíciles de eliminar y, consecuentemente, en un grave problema ambiental. El polietileno y el polipropileno pueden tardar hasta 500 años en descomponerse y actualmente se buscan materiales alternativos que sean más amigables con el medio ambiente (Valdivieso et al., 2013; Emadian et al. 2017). Una opción para sustituir parcialmente el uso de fibras sintéticas son las fibras naturales, estas son biodegradables y de fácil obtención. Además, su uso apoya el trabajo de muchos campesinos en Colombia, convirtiéndose en fuente de ingreso de muchas familias de diferentes regiones de Colombia. Una de las fibras más utilizadas es la fibra de fique (Furcraea bedinghausii), con una producción en Colombia de aproximadamente $300.000 \mathrm{t}$ al año (Ovalle, Blanco y Combariza, 2018), de su cultivo se ocupan y benefician alrededor de 18000 familias específicamente en el Departamento del Cauca y Nariño (Ramirez et al., 2016).

El interés en las fibras naturales está creciendo rápidamente ya que son renovables, baratas y biodegradables. Plantas como el lino (Linum usitatissimum), algodón (Gossypium herbaceum), cáñamo (Cannabis sativa), yute (Corchorus capsularis), sisal (Agave sisalana), bambú (Bambuseae), plátano (Musa paradisiaca), entre muchas otras fuentes de fibras celulósicas, se aplican cada vez más en investigación como refuerzo de compuestos o uso sustitutos de otros materiales. Su disponibilidad, renovabilidad, baja densidad y precio, así como sus destacables propiedades mecánicas, las convierten en una alternativa más ecológica que las fibras de vidrio, carbono y fibras sintéticas (Chegdan y El Mansori, 2018; Elanchezhian et al., 2018). Con el propósito de estudiar el comportamiento de fibras naturales y sintéticas podrían utilizarse modelos matemáticos que permitan estimar la cinética de degradación de sus propiedades en diferentes condiciones. En este sentido los modelos empíricos ofrecen una alternativa para el estudio de procesos donde intervienen múltiples factores y los fenómenos involucrados son complejos de representar desde un enfoque mecanicista o teórico (Pantoja et al., 2016).

El presente trabajo tuvo como objetivo evaluar el comportamiento de las propiedades mecánicas (carga máxima, elongación y módulo de Young) en fibras de fique, plátano y algodón en comparación de la fibra de polipropileno bajo condiciones ambientales en las cuales se desarrolla el cultivo de arveja para plantear un posible remplazo de la fibra sintética que es empleada en el soporte vertical del cultivo.

\section{MATERIALES Y MÉTODOS}

Se utilizaron tres tipos de fibras naturales: fique, plátano y algodón. Como referencia se utilizó la fibra de polipropileno que es la usada actualmente en el tutorado de arveja (figura 1). Las fibras naturales fueron extraídas, desfibradas y secadas para posteriormente ser trenzadas por los campesinos de la región. Por su parte la fibra de polipropileno se obtuvo en centros de acopio locales.

Las fibras se sometieron a diferentes pruebas para conocer sus características físicas, se determinó el diámetro de las fibras utilizando un Stereo microscopio (Discovery V8. Zeizz). La densidad real se determinó empleando el método usado por Cerón et al. (2015), en el cual se utilizó etanol al 99,8\% con densidad de $0,790 \mathrm{~g} / \mathrm{mL}$ a $20^{\circ} \mathrm{C}$ y un picnómetro de $50 \mathrm{~mL}$. El porcentaje de humedad se midió utilizando un determinador electrónico (Versión 1.1, Kern DBS, KERN y Sohn GmbH, Alemania), en el cual se empleó $100 \pm 0.01 \mathrm{~g}$ de muestra. Las pruebas composicionales fueron realizadas en los laboratorios especializados de la Universidad de Nariño por los métodos Van Soest Secuencial Oxidación KMnO4 para el caso de lignina, y Van Soest Secuencial para el caso de celulosa y hemicelulosa y en todos los casos por técnicas Gravimétricas (Viel et al., 2018). 


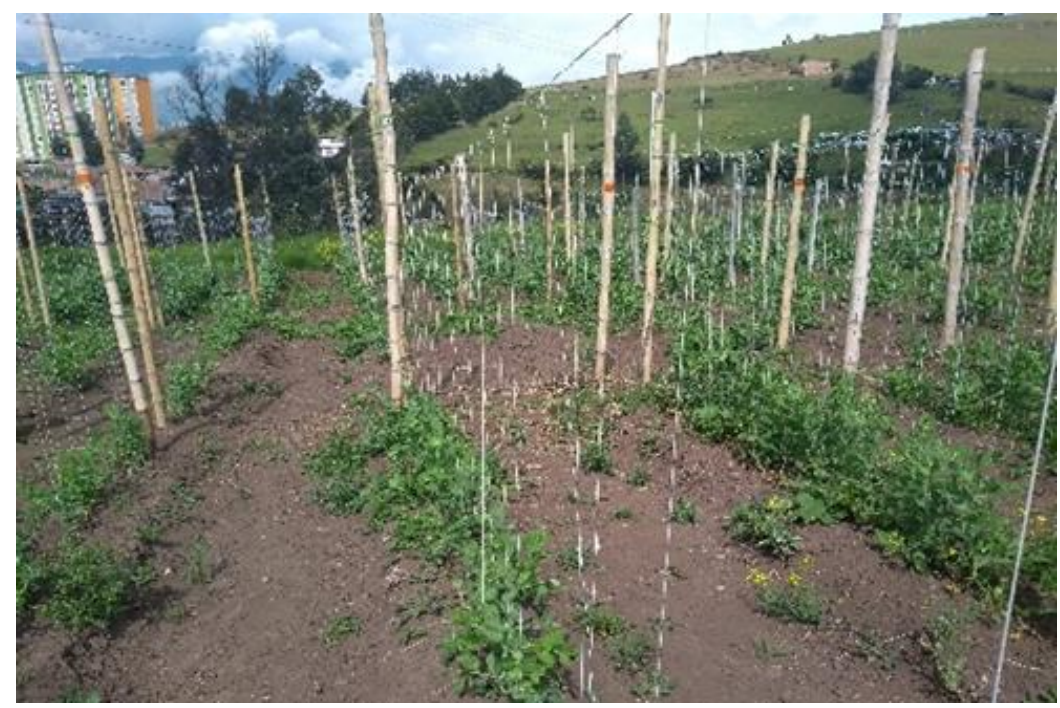

Fig. 1: Fibras utilizadas en tutorado

\section{Propiedades Mecánicas}

Se usó un texturómetro (LS1, LLOYD, USA) al cual se le incorporó unas mordazas de agarre especiales para fibras, se evaluó la carga máxima, elongación y Módulo de Young según la metodología descrita por Mina (2012), siguiendo la norma ASTM D-3822-07 en la cual se sujetan $100 \mathrm{~mm}$ de cada fibra a las mordazas del equipo. Las pruebas mecánicas se realizaron por triplicado a una velocidad de $5 \mathrm{~mm} / \mathrm{min}$, rango de carga de $1000 \mathrm{~N}$, pre-carga de $1 \mathrm{~N}$ y longitud entre mordazas de $100 \mathrm{~mm}$.

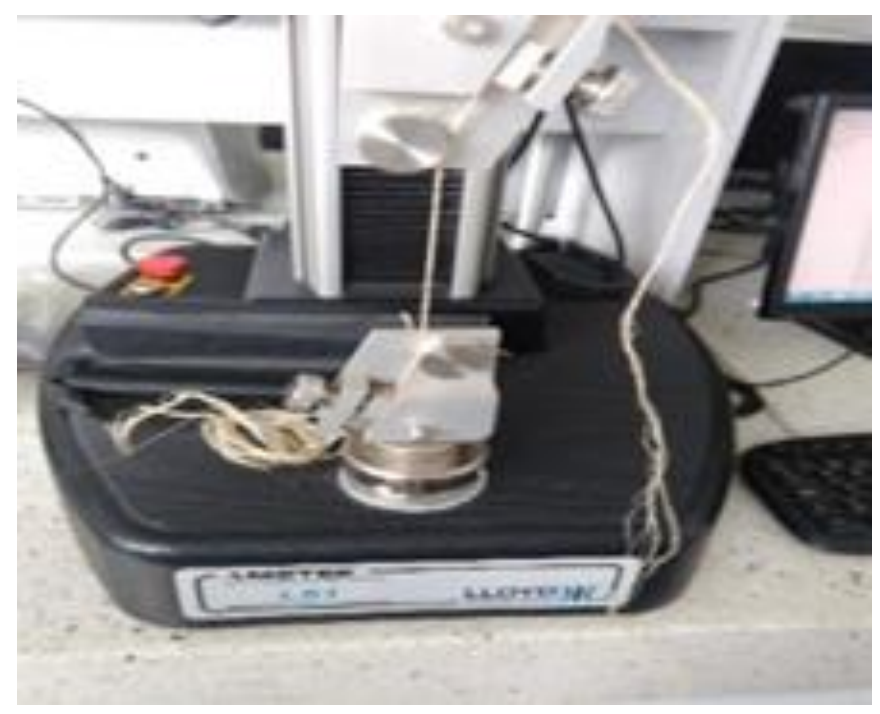

Fig. 2: Pruebas mecánicas

\section{Experimentación en campo}

Se realizaron dos tipos de experimentaciones, la primera como método de observación con pesos adheridos a las fibras y la segunda sin pesos, en las instalaciones del servicio nacional de aprendizaje (SENA), localizado a $2527 \mathrm{msnm}$, a una temperatura promedio de $14^{\circ} \mathrm{C}$ y una humedad relativa promedio de $70 \%$.

Como método de observación se sometieron las fibras con pesos de 1, 2 y $3 \mathrm{~kg}$ con el objetivo de estimar los días de resistencia de cada fibra hasta la ruptura. Las fibras se cortaron de $1 \mathrm{~m}$ de longitud y se suspendieron de un tensor con los pesos establecidos bajo las condiciones climáticas nombradas anteriormente. Se realizó un seguimiento diario para determinar el tiempo requerido para que la fibra fallara y se desprendiera el peso. Con respecto a las fibras sin pesos, estas fueron cortadas con una longitud de $200 \mathrm{~mm}$ y sus extremos fueron atados a unos tensores. Cada 15 días se realizaron las pruebas mecánicas descritas anteriormente y se determinó la pérdida de calidad bajo condiciones ambientales, sin pesos, durante 3 meses. 


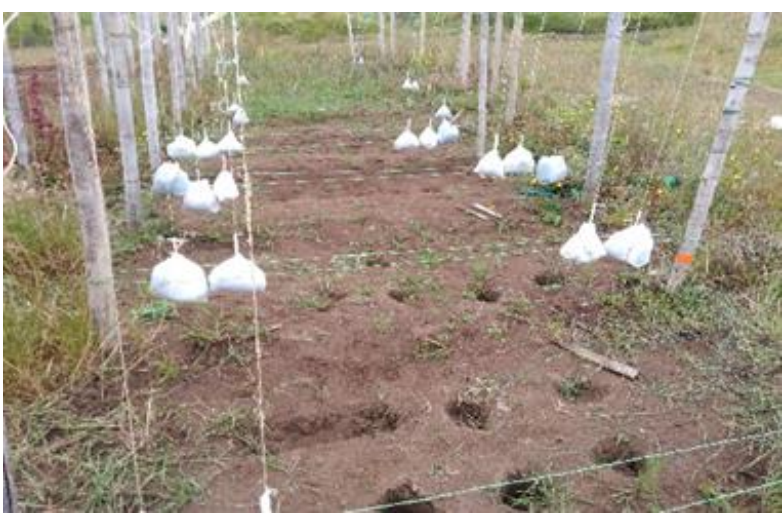

Fig. 3. Experimentación con pesos

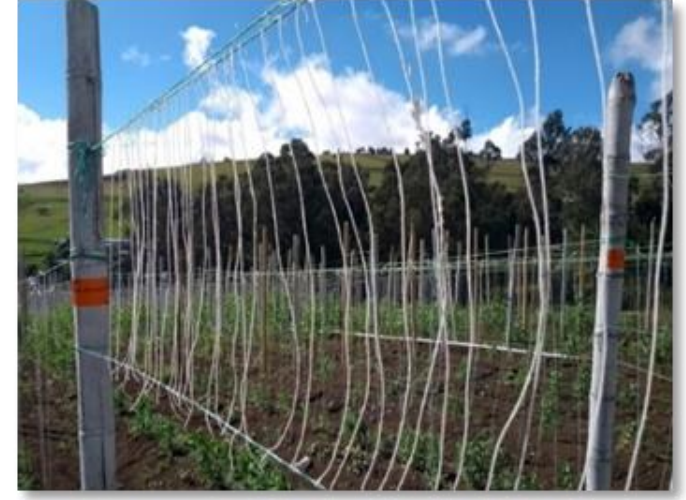

Fig. 4. Experimentación sin pesos

\section{Modelamiento matemático}

En la representación matemática de la pérdida de calidad de las propiedades mecánicas se utilizó el modelo de Page (ecuación 1) del cual se obtuvo los parámetros $k$ y $n$ que permiten caracterizar la cinética de degradación.

$$
\frac{\text { prop }}{\text { prop0 }}=\exp \left(\mathrm{kt}^{\mathrm{n}}\right)
$$

donde prop es la propiedad escogida (módulo de Young, carga máxima o elongación) y prop0 es la dicha propiedad a tiempo inicial. Por lo tanto, prop/prop0 representa el cambio de la propiedad respecto de su valor inicial.

Los índices de ajuste utilizados fueron el coeficiente de determinación ajustado (ecuación 2) y error medio relativo (ecuación 3).

$$
R_{\mathrm{adj}}^{2}=1-\left(1-R^{2}\right)\left(\frac{N-1}{N-m}\right)
$$

Donde $R 2_{\text {adj }}$ corresponde al coeficiente de determinación ajustado, $R^{2}$ corresponde al coeficiente de determinación, $\mathrm{m}$ es el número de parámetros del modelo y $\mathrm{N}$ es el número de observaciones. Según Villa et al. (2015), el parámetro $R^{2}$ adj es un estadístico que considera la variación de los datos experimentales por medio del coeficiente de determinación, y el número de parámetros por cada modelo matemático.

$$
\mathrm{MRE}=\frac{100}{\mathrm{~N}} \sum_{\mathrm{i}=1}^{\mathrm{N}}\left(\frac{\text { prop }_{\text {exp }, \mathrm{i}}-\text { prop }_{\text {pre }, \mathrm{i}}}{\text { prop }_{\text {exp }, \mathrm{i}}}\right)
$$

donde MRE es el error medio relativo (\%), $\mathrm{N}$ es el número de observaciones, propexp es el valor i-ésimo de la propiedad experimental y prop pre es el valor i-ésimo de la propiedad predicha.

Los parámetros $\mathrm{k}$ y $\mathrm{n}$ del modelo de Page para cada propiedad fueron estimados usando un método de regresión no lineal de la herramienta estadística Matlab® R2015b (The MathWorks Inc.,Natick, MA, USA).

\section{RESULTADOS Y DISCUSIÓN}

Los resultados de la tabla 1 indican que los valores de diámetro utilizados son cercanos a los reportados por Muñoz, Hidalgo y Mina (2014) con 0,87 mm para fibras de fique y plátano para refuerzo de plásticos, así mismo los valores de densidad para cada fibra encontrados fueron muy cercanos a los reportados por el mismo autor para estudios con fibra de fique y los reportados para fibra de plátano de 0,8 ( $/ \mathrm{ml})$ por Gutiérrez et al. (2005).

Estrada (2010) reporta valores de humedad que oscilan entre 8 y 10\% para fibras de bambú y algodón, así mismo Córdoba et al. (2010) reportan valores de 7\% de humedad para fibras de tetera empleadas en Nariño para elaboración de artesanías. 
Tabla 1: Caracterización física de las fibras utilizadas en la experimentación.

\begin{tabular}{|l|c|c|c|}
\hline \multicolumn{1}{|c|}{ Fibra } & Diámetro $(\mathrm{mm})$ & Densidad real $\left(\mathrm{g} / \mathrm{cm}^{3}\right)$ & Humedad (\%) \\
\hline Fique & $1,078 \pm 0,05$ & $0,760 \pm 0,10$ & $12,3 \pm 0,2$ \\
\hline Plátano & $1,035 \pm 0,11$ & $0,696 \pm 0,14$ & $12,6 \pm 0,1$ \\
\hline Algodón & $0,987 \pm 0,01$ & $1,133 \pm 0,13$ & $5,8 \pm 0,1$ \\
\hline Polipropileno & $0,938 \pm 0,09$ & $0,761 \pm 0,14$ & $6,2 \pm 0,1$ \\
\hline
\end{tabular}

Según la prueba de composición mostrada en la tabla 2 la celulosa es el componente predominante en las fibras naturales y son resultados similares a los reportados por Muñoz, Hidalgo y Mina (2014) para fibras de fique, este componente otorga en mayor medida la resistencia mecánica de las fibras. La fibra de algodón tiene mayor porcentaje de celulosa que el resto de las fibras por lo que podría ser más resistente, sin embargo, las fibras de fique y plátano tienen en su composición un mayor porcentaje de lignina y hemicelulosa lo que les confiere una textura diferente a la fibra de algodón.

Tabla 2: Caracterización composicional de las fibras naturales utilizadas en la experimentación.

\begin{tabular}{|c|c|c|c|}
\hline Fibras & Celulosa (\%) & Hemicelulosa (\%) & Lignina (\%) \\
\hline Fique & 44,65 & 12,44 & 11,85 \\
\hline Plátano & 60,87 & 10,76 & 17,87 \\
\hline Algodón & 95,09 & 1,15 & 0,44 \\
\hline
\end{tabular}

\section{Experimentación con pesos}

Como se observa en la tabla 3 la determinación de la resistencia a tensión en función del tiempo de cada fibra tuvo como objetivo seleccionar la fibra con mejores propiedades a tensión expuesta a las condiciones ambientales del cultivo, donde una planta de arveja puede llegar a pesar entre 1 y $2 \mathrm{~kg}$ aproximadamente. Esta experimentación se realizó con cargas entre 1 y $3 \mathrm{~kg}$. Los resultados determinaron que la fibra que más días soportó con $1 \mathrm{~kg}$ de peso fue la fibra de polipropileno que es la usada actualmente, y la fibra natural que más días soportó fue la fibra de algodón con 165 días, la fibra de plátano soportó un promedio de solamente 24 días siendo la fibra que menos resistió, a medida que el peso se fue incrementando los días de resistencia de cada fibra disminuyeron. Según Palechor et al. (2016) esta pérdida de fuerza mecánica es el resultado de la exposición al calor, luz solar, humedad y/o enzimas que cortan y debilitan las cadenas poliméricas, además, los materiales hidrofílicos presentan cambios importantes en sus propiedades mecánicas cuando entran en ambientes húmedos.

Tabla 3: Días de resistencia registrados para cada fibra natural y sintética en el ensayo de experimentación con pesos

\begin{tabular}{|l|c|c|c|}
\hline \multirow{2}{*}{ Fibra } & \multicolumn{3}{c|}{ Peso } \\
\cline { 2 - 4 } & $1 \mathrm{~kg}$ & $2 \mathrm{~kg}$ & $3 \mathrm{~kg}$ \\
\hline Polipropileno & $210 \pm 12$ & $91 \pm 12$ & $76 \pm 13$ \\
\hline Algodón & $165 \pm 13$ & $80 \pm 13$ & $61 \pm 10$ \\
\hline Fique & $63 \pm 9$ & $22 \pm 7$ & $10 \pm 2$ \\
\hline Plátano & $24 \pm 2$ & $10 \pm 1$ & $2 \pm 1$ \\
\hline
\end{tabular}

\section{Estudio de propiedades mecánicas}

Teniendo en cuenta que la finalidad de las fibras en este estudio es poder soportar los tallos trepadores de la planta de arveja durante el ciclo de vida del cultivo, se estudiaron tres propiedades mecánicas (carga máxima, elongación y Módulo de Young) para las cuales se determinó su comportamiento en los días de estudio y la bondad de ajuste de los datos experimentales al modelo de Page.

\section{Carga máxima}

En la figura 5 se puede observar la variación de la carga máxima respecto al tiempo, los valores de esta propiedad disminuyen al transcurrir los días de experimentación. La fibra de plátano fue la que demostró tener más capacidad de carga al inicio de la experimentación, pero también fue la fibra que mostro una mayor pérdida de calidad de esta propiedad en comparación con el resto de las fibras. Tal como manifiesta Suvendu et al. (2013), la ganancia de humedad de las fibras lignocelulósicas generalmente asciende a varias veces su peso seco cuando están expuestas a ambientes húmedos. La hinchazón que resulta de la absorción de agua expone las cadenas de celulosa, un elemento esencial constituyente de las fibras, a microorganismos que degradan la celulosa. Esto, a su vez, conduce a una rápida y significativa pérdida de fuerza. 


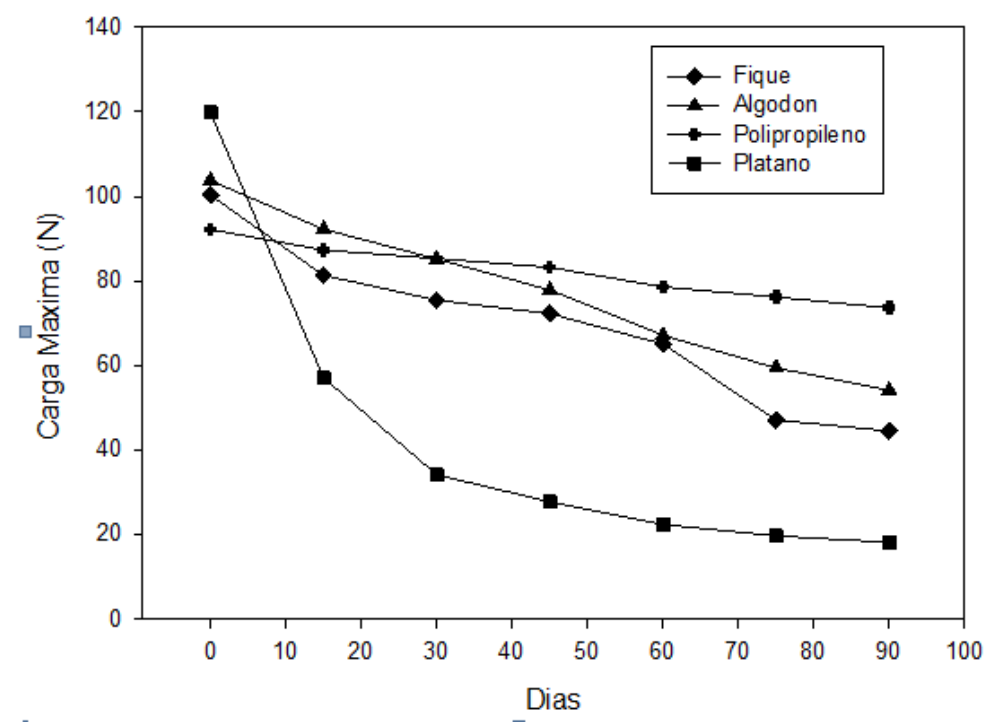

Fig. 5: Cinética del comportamiento de carga máxima

En la tabla 4 se puede observar que los valores de $R^{2}$ adj y MRE presentaron valores que indican la bondad de ajuste de los datos experimentales al modelo empírico evaluado. El coeficiente de determinación ajustado presentó valores mayores e iguales a 0.97 en la mayoría de los resultados, aspecto destacado por Kocayigit y Doymaz (2011). Del mismo modo se obtuvo MRE inferiores al 10\%, resultado que, para Correa et al. (2011), demuestra un buen ajuste a los datos experimentales. El valor de $k$ indica la velocidad máxima de pérdida de calidad mecánica (o tasa de degradación exponencial) que tiene la propiedad de una fibra natural o sintética. Un valor del parámetro $n>1$ indica que $k$ aumenta con el tiempo; cuando $n<1, k$ disminuye con el tiempo; y si $\mathrm{n}$ toma valores cercanos o aproximadamente iguales a $1, \mathrm{k}$ podría considerarse constante con el tiempo.

Según las constantes de velocidad de pérdida de calidad ( $k$ ) relacionadas con la carga máxima (tabla 4), la fibra de plátano presentó una mayor velocidad de degradación $\left(k=-0,20\right.$ días $\left.^{-1} ; n=0,52\right)$ respecto a las demás fibras evaluadas. Las fibras de fique, algodón y polipropileno no presentaron diferencias estadísticamente significativas entre los valores de $\mathrm{k}$. Los resultados mostraron que la fibra de plátano mantuvo su capacidad de carga por menos tiempo respecto a las otras fibras evaluadas. La resistencia de las demás fibras puede ser explicada por la cantidad de celulosa que forma su estructura que, de acuerdo con Muñoz et al. (2014), es de aproximadamente $70 \%$ para las fibras de fique y plátano y de casi $95 \%$ para la fibra de algodón. La celulosa aportaría la mayor parte de la resistencia mecánica a estas fibras (Velásquez, Pelaéz, y Giraldo, 2016).

Tabla 4: Parámetros cinéticos del comportamiento de la propiedad de carga máxima para cada fibra utilizada

\begin{tabular}{|l|c|c|c|c|}
\hline \multicolumn{1}{|c|}{ Fibra } & $\mathrm{k}\left(\mathrm{dí}^{-1}\right)$ & $\mathrm{n}$ & $\mathrm{R}^{2} \mathrm{adj}$ & $\mathrm{MRE}$ \\
\hline Fique & $-0,011 \pm 0,003^{\mathrm{a}}$ & $0,943 \pm 0,071$ & 0,93 & $5,4 \%$ \\
\hline Plátano & $-0,204 \pm 0,094^{\mathrm{b}}$ & $0,526 \pm 0,110$ & 0,99 & $2,3 \%$ \\
\hline Algodón & $-0,007 \pm 0,007^{\mathrm{a}}$ & $1,081 \pm 0,228$ & 0,98 & $1,9 \%$ \\
\hline Polipropileno & $-0,006 \pm 0,006^{\mathrm{a}}$ & $1,111 \pm 0,696$ & 0,97 & $1,5 \%$ \\
\hline
\end{tabular}

\section{Módulo de Young}

En la figura 6 se observa una tendencia decreciente de los valores al trascurrir el tiempo de experimentación. Al inicio de las pruebas la fibra de plátano y fique presentaron mayores valores que las fibras de algodón y polipropileno, esto pudo darse por un porcentaje mayor de lignina y hemicelulosa en la estructura de estas fibras, lo que conlleva a un cambio en su rigidez. Según Sergio, Blanco, y Combariza (2018) la disminución o afectación de las propiedades mecánicas de una fibra está determinada por el ambiente al cual están sometidas involucrando factores químicos, físicos, mecánicos y biológicos. Las fibras estuvieron sometidas a condiciones ambientales, tales como luz, lluvias, viento, y esta interacción deteriora la composición y estructura del material y, en consecuencia, sus propiedades mecánicas. 


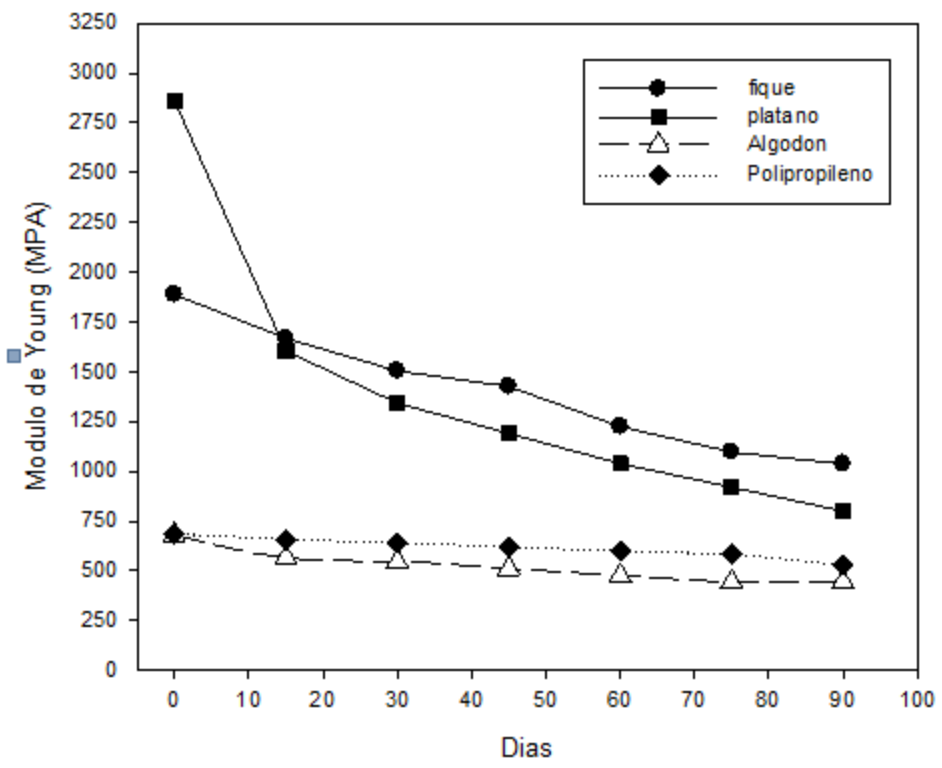

Fig. 6: Cinética del comportamiento del Módulo de Young

En la tabla 5 y no se evidencian diferencias significativas entre las constantes de velocidad de las fibras de fique y algodón evaluadas, no obstante, la fibra de plátano presentó mayor velocidad de pérdida mecánica con diferencias significativas respecto a las fibras de fique, algodón y polipropileno, además el parámetro indica que su velocidad disminuye al transcurrir el tiempo. La menor pérdida mecánica se observó en la fibra de polipropileno. Después de su uso en el tutorado de arveja, este tipo de fibra constituye un contaminante ambiental para el cual se busca un sustituto biodegradable, que puede ser una fibra como el algodón o el fique que soporten el cultivo y luego se deterioren rápidamente para hacer parte de la materia orgánica dentro del sistema productivo.

Tabla 5: Parámetros cinéticos del comportamiento del Módulo de Young para cada fibra utilizada

\begin{tabular}{|l|c|c|c|c|}
\hline \multicolumn{1}{|c|}{ Fibra } & $k\left(\right.$ día $\left.^{-1}\right)$ & $n$ & $R^{2}$ & $M R E$ \\
\hline Fique & $-0,0128 \pm 0,01^{\mathrm{b}}$ & $0,970 \pm 0,086$ & 0,98 & $3,4 \%$ \\
\hline Plátano & $-0,1732 \pm 0,17^{\mathrm{a}}$ & $0,435 \pm 0,022$ & 0,99 & $4,3 \%$ \\
\hline Algodón & $-0,0421 \pm 0,04^{\mathrm{b}}$ & $0,541 \pm 0,325$ & 0,97 & $2,9 \%$ \\
\hline Polipropileno & $-0,0008 \pm 0,001^{\mathrm{c}}$ & $1,261 \pm 0,789$ & 0,95 & $1,8 \%$ \\
\hline
\end{tabular}

\section{Propiedad de elongación}

En la figura 7 se observa que las fibras de algodón y polipropileno registraron un porcentaje de deformación aproximadamente del $40 \%$, indicando que este tipo de materiales son muy flexibles en comparación con las fibras de fique y plátano que presentaron valores bajos, esta diferencia está determinada por la rigidez del material, cuando mayor es la elongación máxima menor es la rigidez, esto se puede comprobar en el parámetro de módulo de Young explicado anteriormente. Valores altos de elongación podría ocasionar problemas en el momento de utilizar la fibra en campo ya que a medida que la planta tutorada va creciendo y ganando peso necesita un soporte que la mantenga estable y si la fibra se estira demasiado la planta podría caer al suelo y verse afectada.

La pérdida de calidad del parámetro de elongación según puede estar atribuida a la absorción o contacto con agua líquida o vapor, esta agua penetra en los capilares y en los poros, y difunde dentro de las regiones amorfas, reduciendo la flexibilidad o elongación. Ambientes húmedos causan una menor resistencia de las propiedades mecánicas (Rodríguez y Sarache, 2014).

En la tabla 6 se muestran los parámetros cinéticos para la propiedad de elongación para fibras evaluadas. Las fibras de fique y polipropileno no presentaron diferencias estadísticamente significativas, lo mismo ocurrió entre las fibras de plátano y algodón que obtuvieron un parámetro $n$ de 0,8 , lo cual significa que su velocidad de pérdida mecánica disminuye al transcurrir el tiempo. 


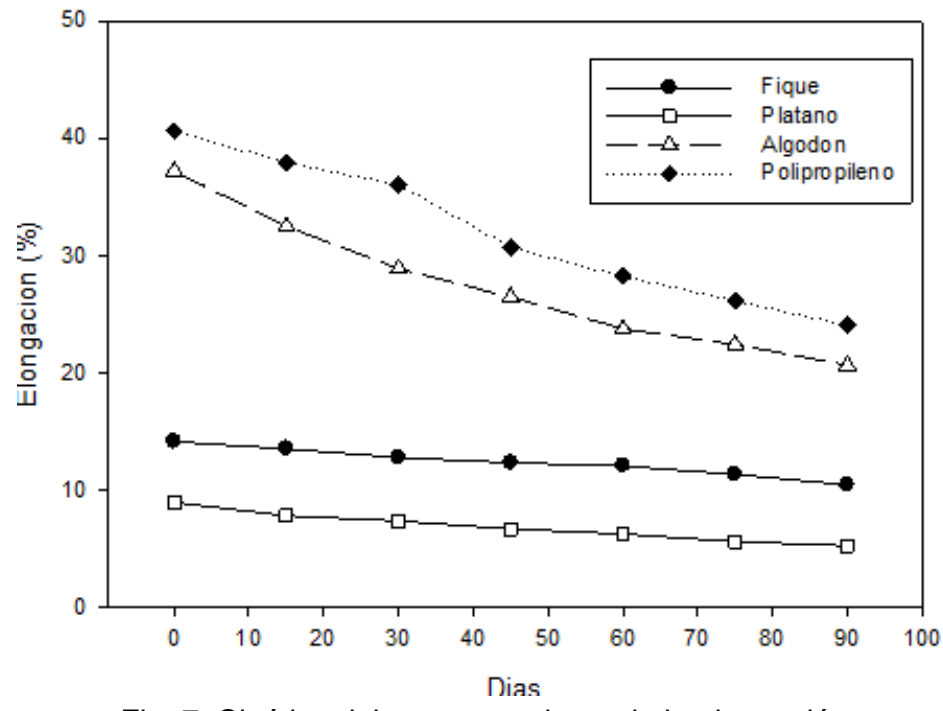

Fig. 7: Cinética del comportamiento de la elongación

Tabla 6: Parámetros cinéticos del comportamiento de elongación para cada fibra utilizada

\begin{tabular}{|l|c|c|c|c|}
\hline \multicolumn{1}{|c|}{ Fibra } & $k\left(\right.$ día $\left.^{-1}\right)$ & $n$ & $R^{2}$ & $M R E$ \\
\hline Fique & $-0,0039 \pm 0,004^{\mathrm{ab}}$ & $1,060 \pm 0,086$ & 0,96 & $3,9 \%$ \\
\hline Plátano & $-0,0148 \pm 0,010^{\mathrm{b}}$ & $0,866 \pm 0,032$ & 0,97 & $3,3 \%$ \\
\hline Algodón & $-0,0169 \pm 0,006^{\mathrm{b}}$ & $0,800 \pm 0,235$ & 0,99 & $2,1 \%$ \\
\hline Polipropileno & $-0,0040 \pm 0,004^{\mathrm{ab}}$ & $1,178 \pm 0,963$ & 0,95 & $1,2 \%$ \\
\hline
\end{tabular}

Es claro que las fibras naturales pueden ser una buena alternativa como soporte vertical en de sistemas agrícolas, sin embargo las pruebas realizadas indican claramente que la resistencia de ellas en términos de carga y en función del tiempo aun no es comparable con el polipropileno, sin embargo los resultados muestran algunos resultados interesantes que podrán mejorarse con el uso de alternativas tecnológicas como el uso de recubrimientos biopoliméricos, los cuales podrían aportar mayor resistencia en tiempo y fuerza para los sistemas de tutorado estudiados.

\section{CONCLUSIONES}

De acuerdo a los resultados de este estudio, y de su discusión y análisis se pueden extraer las siguientes conclusiones:

i) El modelo utilizado para simular el proceso de pérdida de calidad de las fibras mostró una buena bondad de ajuste a los datos experimentales; (ii) Las propiedades mecánicas evaluadas sufrieron una pedida de calidad considerable durante los días de experimentación; (iii) La fibra natural de plátano mostró un deterioro y pérdida de calidad mecánica mayor que el resto de las fibras así como una elevada velocidad máxima de pérdida en la mayoría de las propiedades evaluadas por lo tanto podría ser descartada como posible remplazo de la fibra de polipropileno; y (iv) Las fibras de fique y algodón estadísticamente mostraron un comportamiento mecánico similar por lo cual podrían ser probadas directamente en el cultivo como alternativa al tutorado con polipropileno.

\section{AGRADECIMIENTOS}

Agradecimientos al sistema general de regalías - SGR (Colombia) por la financiación del proyecto de "Investigación para el mejoramiento de la tecnología de producción de arveja (Pisum sativum L.) en el departamento de Nariño" proyecto - Cod. BPIN 2013000100278, y a los grupos de investigación Gaida y Cultivos Andinos de la Universidad de Nariño por su apoyo en laboratorio y campo.

\section{REFERENCIAS}

American Society Testing For Materials - ASTM. Standard Test Method for Tensile Properties of Single Textiles Fibers, ASTM D-3822, (2007) 
Cerón, A., L. Latorre y otros cuatro autores, Determinación de Constantes de Velocidad de Rehidratación y Cambios sobre algunas Propiedades Físicas en Semillas de Arveja (Pisum Sativum L.), Revista Lasallista de Investigación, 12 (1), 21-32 (2015)

Chegdan, F. y M. Mansori, Friction Scale Effect in Drilling Natural Fiber Composites, doi: https://doi.org/10.1016/j.triboint.2017.12.006, Tribology International, 119 (622-630) (2018)

Córdoba, C., J. Mera y D. Martínez, Aprovechamiento de Polipropileno y Polietileno de Alta Densidad, Reforzados Con Fibra Vegetal, Tetera (Stromanthe Stromathoides), Revista Iberoamericana de Polímeros, 11 (7), 417-27 (2010)

Correa, P.C., F. Mendes, G. Henrique y H. Oliveira, Mathematical Modeling of the Drying Process of Corn Ears, doi:10.4025/actasciagron.v33i4.7079, Acta Scientiarum Agronomy, 33 (4), 575-81 (2011)

Elanchezhian, C., B. Vijaya Ramnath y otros cuatro autores, Review on Mechanical Properties of Natural Fiber Composites, doi:10.1016/j.matpr.2017.11.276, Materials Today Proceedings, 5(1), Elsevier Ltd, 1785-90 (2018)

Emadian, S.M, T.T. Onay y B. Demirel, Biodegradation of bioplastics in natural environments, http://dx.doi.org/10.1016/j.wasman.2016.10.006, Waste Management, 59, 526-536 (2017)

Estrada, M., Extraccion y Caracterizacion Mecanica de Las Fibras de Bambu (Guadua Angustifolia) Para su Uso Potencial Como Refuerzo de Materiales Compuestos (2010)

Forero, A. y G. Ligarreto, Evaluacion de Dos Sistemas de Tutorado para el Cultivo de la Arveja Voluble (Pisum Sativum L.) En Condiciones de La Sabana de Bogotá, Revista Colombiana de Ciencias Agrícolas, (3) 81-94 (2009)

Gutiérrez, I., R. Zuluaga, J. Cruz y P. Gañán, Influencia del Tratamiento con Vapor Sobre la Estructura y Comportamiento Físico-Mecánico de Fibras de Plátano, Información Tecnológica, 16(2), 15-21 (2005)

Kocayigit, F. e I. Doymaz, Drying and Rehydration Behaviors of Convection Drying of Green Peas doi:10.1080/07373937.2011.591713, Drying Technology, 29 (11), 1273-82 (2011)

Mina, J., Caracterización físico-mecánica de un almidón termoplástico (tps) de yuca y análisis interfacial con fibras de fique, Biotecnologia en El Sector Agropecuario y Agroindustrial, 10 (2) 99-109 (2012)

Muñoz, M., M. Hidalgo y J. Mina, Fibras de fique una alternativa para el reforzamiento de plásticos, Influencia de la modificación superficial, Biotecnología en El Sector Agropecuario y Agroindustrial, 12 (2), 60-70 (2014)

Ovalle, S., C. Blanco y M. Combariza, Exploring the Composition of Raw and delignified Colombian fique fibers Tow and Pulp, doi:10.1007/s10570-017-1599-9, Cellulose, 25 (1), Springer Netherlands, 151-65 (2018)

Palechor, J., A. Ceron, H. Villada y M. Salazar; Deterioro de Una Bolsa Biodegradable de Almidón de Yuca Con Ácido Poliláctico En Un Vivero, Vitae, 23, 585-89 (2016)

Pantoja, D.C., O. Osorio, D.F., Mejía y H. Váquiro, Procesamiento de Arvejas (Pisum Sativum L.) Parte 1: Modelado de La Cinética de Secado por Capa Delgada de Arveja, Variedades Obonuco Andina y Sureña, doi: 10.4067/S071807642016000100009, Información Tecnologica, 27 (1) 69-80 (2016)

Ramirez, A., V. Fernandez y N.E. Sanchez, The Use of Common Duckweed (Lemna Minor) in the Treatment of Wastewater from the Washing of Sisal Fiber (Furcraea Bedinghausii), Ingenieria y Competitividad, 18 (2), 25-34 (2016)

Rodríguez, L. y W. Sarache, Compuestos de Poliéster Reforzados con Fibra de Plátano / Banano (Musa paradisiaca) Modificada Químicamente. Comparación con Fibra de Vidrio y Fique (Furcraea andina), https://doi.org/10.4067/S071807642014000500005, Información Tecnológica, 25(5), 27-34 (2014)

Solarte, R., O. Oswaldo y O. Checa, Importancia del subsector productivo de arveja (phisum sativum) en el departamento de Nariño, Revista Vitae, 23 (S1), 844- 848 (2016)

Suvendu, M., S. Prosenjit y otros tres autores, Enhanced Biodegradation Resistance of Biomodified Jute Fibers, doi:http://dx.doi.org/10.1016/j.carbpol.2012.11.061, Carbohydrate Polymers, 93, 597-603 (2013)

Valdivieso, M., Y. Ortegon e Y. Uscategui, Biopolímeros : avances y perspectivas biopolymers, Dyna 181 (0012-7353), 171-80 (2013)

Velásquez, S., G. Pelaéz y D. Giraldo, Uso de Fibras Vegetales En Materiales Compuestos de Matriz Polimérica: Una Revisión Con Miras a su Aplicación en el Diseño de Nuevos Productos, Informador Técnico, 80, 77-86 (2016)

Viel, M., F. Collet y C. Lanos, Chemical and multi-physical characterization of agro-resources by-product as a possible raw building material, Industrial Crops and Products, 120, 214-237 (2018)

Villa, H., H. Váquiro y J. Telis, The Effect of Power-Ultrasound on the Pretreatment of Acidified Aqueous Solutions of Banana Flower-Stalk : Structural, Chemical and Statistical Analysis, doi:10.1016/j.indcrop.2014.12.022, Industrial Crops y Products 66, Elsevier B.V., 52-61 (2015) 
